\title{
Piet Derksen und seine Medienarbeit: \\ Stein der Weisen oder Stein des Anstoßes?
}

\author{
von Joan Hemels
}

Am 27.06.1990 wurde im Zentrum der europäischen Hauptstadt Brüssel das 'Institut de Journalisme - European Media Studies Robert Schuman' (RobertSchuman-Institut) eröffnet und eingeweiht. ${ }^{1}$ Die Eröffnungszeremonie bestand aus der Enthüllung einer Tafel zum Andenken an Robert Schuman; sie wurde vollzogen von dem Mitglied des Europaparlaments und ehemaligen belgischen Ministerpräsidenten Leo Tindemans, zusammen mit dem niederländischen Unternehmer (i. R.) Piet Derksen. Msgr. De Hovre, Weihbischof von MechelenBrüssel, führte die Einweihung durch; er ist auch Kuratoriumsmitglied des neuen Brüsseler Instituts und Belgiens 'Medienbischof'.

Dorothee Wilms, Ministerin für Innerdeutsche Beziehungen, hielt einen bemerkenswerten Vortrag über Deutschland und Europa auf dem Weg zur Einheit. Der Präsident des polnischen Rundfunks, Andrzej Drawicz, behandelte das aktuelle Thema der neuen Rolle der Medien in Osteuropa. Bei der Feierstunde waren viele Vertreter der internationalen kirchlichen und weltlichen Prominenz, somit führende Fachleute der Medien, anwesend.

\section{Evangelisation als Fernziel des Robert-Schuman-Instituts}

Erkundigt man sich, wie und warum das Robert-Schuman-Institut zustande gekommen ist, dann hört man eigentlich nur einen Namen: Piet Derksen. Recht unauffällig war er mit seiner Gattin an der Eröffnungsfeier beteiligt. Weiter recherchierend entdeckt man nicht ohne Mühe, daß sich derselbe Niederländer in den letzten Jahren als großzügiger Sponsor caritativer und massenmedialer Aktivitäten einen bestimmten Ruf erworben hat. Obwohl nicht immer mit bösen Absichten wird Derksen in ausländischen und niederländischen Veröffentlichungen bequemlichkeitshalber manchmal in die Reihe der bettelnden Fernsehmissionare eingeordnet, die am liebsten weltweit missionieren möchten.

Tatsächlich zielt Derksen mit seiner Medienarbeit auch auf einen 'Satelliten-Jesus', nur darf man ihn nicht im Rahmen der unkeuschen Fehltritte eines Jimmy Swaggart oder der Betrügereien eines Jim Bakker (tatsächlich: mit niederländischen Vorfahren) betrachten. Derksen macht der katholischen Kirche den Vorwurf eines schwachen Sendungsbewußtseins: Sie hätte den Bildschirm nicht den protestantischen Fundamentalisten überlassen dürfen. Bekehrung per Bildschirm mit Massenevangelisation via Satellit als Fernziel sieht Derksen auch als eine Aufgabe der katholischen Laien. Dazu werden zuverlässige, kirchentreue, international orientierte Journalisten gebraucht, und dies erklärt sein Interesse an Weiterbildung für Medienarbeit.

Prof. Dr. Joan Hemels ist Inhaber des Lehrstuhls für Kommunikationswissenschaft, insbesondere Kommunikationsgeschichte, der Studienrichtung Kommunikationswissenschaft der Universität Amsterdam, Niederlande. 
In diesem Aufsatz wird versucht, einen Teil der von Derksen und seiner finanzkräftigen Gruppe entfalteten Tätigkeiten zu beleuchten, nämlich die auf Medienarbeit bezogenen Projekte in den Niederlanden und im Ausland. Nicht alles, was verschleiert ist, läßt sich einfach aufdecken. Dieser Beitrag ist zu verstehen als ein - jedenfalls nicht böse gemeinter - Versuch zur Entmythologisierung einer Person, die oft ins Gerede kommt.

Die Erklärung des Bemühens Derksens um die Verbreitung des Evangeliums und die Bekehrung der Menschheit per Bildschirm auch mit Hilfe des Satellitenfernsehens kann u. a. auf soziologischer Ebene, im Bereich der Änderungen in der niederländischen Gesellschaft und in der katholischen Kirche gesucht werden. Die Entchristlichung und der weitergehende Prozeß der Säkularisierung provozieren einen Einsatz der privaten elektronischen Medien im Dienst der Verkündigung des Wortes Gottes und der Religion, der in Europa noch kaum eine Vorgeschichte hat. Das Predigen über Kabel macht den Bildschirm auch in diesem Teil der Welt immer mehr zur Kanzel. Die Folgen dieser Entwicklung darf man rechtzeitig und kritisch reflektieren.

Die tiefere persönliche Motivation, die Derksen bewegt, seinVermögen u.a. der katholischen Medienarbeit zur Verfügung zu stellen, läßt sich nicht hinter dem Schreibtisch aufdecken. Nur seine eigenen Aussagen in Zeitungsausschnitten und in Veröffentlichungen der von ihm gegründeten 'Stiftung zum Zeugnis der Liebe Gottes' bieten einige Anhaltspunkte. Einmal, nämlich am 05.06.1989, ließ Derksen sich in einer niederländischen Fernsehsendung interviewen. ${ }^{2}$ Die Fernseh-Dokumentation lieferte ein hervorragendes Bild von Derksens Aktivitäten.

\section{Der Studienplan des Robert-Schuman-Instituts}

Das Robert-Schuman-Institut wurde im September 1988 gegründet mit der Aufgabe, 'junge Männer und Frauen zu Medienmenschen auf dem Weg zum Jahre 2000 auszubilden', wie es im farbigen Werbeprospekt heißt. Dieser Studiengang soll sich durch drei Zielsetzungen kennzeichnen: erstens eine praxisbezogene Ausbildung für Fachleute anzubieten, zweitens den Teilnehmern in einer Arbeits- und Freundschaftsgemeinschaft eine europäische Ausbildung zur Verfügung zu stellen, und drittens im Hinblick auf die Entwicklung einer Lebensanschauung in bezug zu Mensch und Gesellschaft eine katholische Ausbildung anzutragen.

Die Satzung des Robert-Schuman-Instituts enthält u. a. diese Grundsatzerklärung: 'Als Journalisten sind sie (die Absolventen) berufen, der Kirche Bekanntheit zu verleihen und den einzigartigen Charakter der Kirche bekannt zu machen in einer Gesellschaft, die durch Skeptizismus und Pluralismus geprägt wird'. 'Im Dienst des Menschen', so lautet die Devise des Instituts, das nach dem Staatsmann, Christen und Europabefürworter Robert Schuman ${ }^{3}$ (geboren am 29.06.1886 in Luxemburg, gestorben am 04.09.1963 in Scy-Chazelles in der Nähe von Metz) benannt wurde. Als Außenminister Frankreichs machte er am 09.05.1950 den Vorschlag zur deutsch-französischen Versöhnung und legte dadurch den Grundstein für ein vereintes Europa. Im Juni 1990 wurde das Verfahren zur Seligsprechung Schumans eingeleitet. 
Das Robert-Schuman-Institut ist ein Privatinstitut mit Internatscharakter: Die jährlich ungefähr 20 Studentinnen und Studenten aus verschiedenen europäischen Ländern wohnen als Jahrgangsgruppe im Institutsgebäude. Nicht nur hervorragend ausgestattete Seminarräume, Hörfunkstudio, Videoraum und Lehrredaktion mit der modernsten Apparatur für elektronische Textverarbeitung, sondern auch eine Kapelle wechseln sich mit Wohnräumen in dem gründlich renovierten Institutsgebäude ab. Der Studiengang dauert insgesamt 10 Monate und zielt auf journalistische Arbeit bei Printmedien, Hörfunk und Fernsehen. Die 20.Teilnehmer des zweiten Jahrgangs 1989/90 vertraten insgesamt 11 Nationalitäten. Sie hatten vorher ein Universitäts- oder Hochschulstudium absolviert und zahlen für das Ergänzungsstudium ca. 1.400,- DM.

Die Dozenten kommen aus verschiedenen Ländern Europas; auch sie können durch die drei schon erwähnten Stichwörter charakterisiert werden: professionell, europäisch und christlich. In den Übungen wurde schon eine ganze Anzahl von Medienprodukten fertiggestellt: ein 80seitiges Magazin 'Terres d'Europe' über Landwirtschaft für die Europäische Gemeinschaft, als Sonderausgabe 'features. Un magazine pour l'Europe' in fünf Sprachen, die Probenummer eines Sonntagsblatts mit dem Titel 'Dimanche Actualite' sowie Hörfunkund Videoproduktionen. Die Institutszeitschrift 'Carrefours. A journal of news and features by the European Media Studies' bietet den Studierenden die Möglichkeit, mit Hilfe der zuständigen Dozenten das Handwerk des Zeitungmachens zu lernen. Der praxisbezogene Teil des Ausbildungsprogramms ist, so darf man feststellen, beeindruckend und fördert zweifelsohne die Fähigkeit, multimedial, d. h. für Printmedien, Hörfunk und Fernsehen zu arbeiten. Gerade dieser Aspekt der vielseitigen Ausbildung muß meines Erachtens in Hinblick auf die Umwälzung der Medienlandschaft und im Rahmen der beruflichen Mobilität innerhalb der Kommunikationsberufe als sehr positiv bewertet werden.

\section{Hinter den Kulissen eines Freizeitgestaltungsimperiums}

Im Vergleich zu amerikanischen Verhältnissen sind Stiftungen privater Ausbildungsinstitute für Kommunikationsberufe in Europa selten. Die Art und Weise der Finanzierung und der Gründung des Robert-Schuman-Instituts mutet tatsächlich fast amerikanisch an. Dies gilt auch für den eigentlichen Initiator des Instituts, den die Phantasie mancher Journalisten anregenden niederländischen Multimillionär Piet Derksen (1990 wurde er 77 Jahre alt) und seine Frau Trude Derksen-Nefkens (69). Wegen großer Verdienste um die Kirche wurden beide Anfang 1990 von Papst Johannes Paul II. ausgezeichnet: Derksen wurde zum Kommandeur des Heiligen Gregoriusorden ernannt, und seine Frau bekam die Auszeichnung 'Pro Ecclesia et Pontifice'.

Das Ehepaar Derksen gehört zum Vorstand des eingetragenen Vereins 'Stichting Getuigenis van Gods Liefde' (Stiftung zum Zeugnis der Liebe Gottes) in Eindhoven, Niederlande. Diese Stiftung wird finanziert aus Mitteln der von Derksen gegründeten 'Stichting Levend Water Beheer' (Stiftung Lebendiges Wasser Verwaltung). Der Geschäftsführer der Zeugnis der Liebe Gottes-Organisation, der Niederländer Willy Boers, ist auch Vorsitzender des Kuratoriums ('Board of Administration') des Robert-Schuman-Instituts. Piet Derksen ist Präsi- 
dent der Stiftung zum Zeugnis der Liebe Gottes und Mitglied des Kuratoriums des Robert-Schuman-Instituts.

Nach einer schweren Krankheit und einer als wunderbar erfahrenen Genesung änderte sich das Leben für Piet Derksen tiefgreifend. Das Ehepaar machte eine Pilgerfahrt nach Lourdes und lernte in Paray-le-Monial (Frankreich) die französische charismatische Emmanuel-Bewegung kennen, die 1989 auch in den Niederlanden kirchlich anerkannt wurde und der Derksen sehr zugetan ist. Die 300 Kernsympathisanten zählende Bewegung bildet das geistige Fundament der von Derksen und seiner Frau ins Leben gerufenen Organisationen. In Paray-le-Monial erlebten beide eine Wiedergeburt, wie sie später erklärten.

Piet Derksen und seine Gattin waren schon Multimillionäre, als sie 1982 ihr herrschaftliches Landhaus im belgischen Turnhout verkauften; ihre Kunstschätze boten sie bei Christies an. Zusammen mit den Privatanteilen (jedenfalls $65 \%$ ) des Unternehmens des Ehepaars wurden die Einnahmen aus dem Verkauf der Villa und des Kunstbesitzes (1 Mio. Gulden) in die 'Stiftung Lebendiges Wasser Verwaltung' untergebracht. Die Stiftung verwaltete außerdem die an der Börse gehandelten Anteile eines Unternehmens mit dem Namen 'Center Parcs', dessen Expansion durch dieselbe Stiftung finanziert wurde; schließlich flossen die Erträge der Anteile zur Stiftung zurück. Das Kuratorium zählte drei Mitglieder: das Ehepaar Derksen und Schwiegersohn Pieter Terwan. Als neuen Wohnort wählten Piet und Trude ein Ferienhaus in einem (eigenen) Feriendorf in der Nähe von Valkenswaard, nicht weit entfernt von Eindhoven. Die 'Stiftung Lebendiges Wasser Verwaltung' zahlte den vier Kindern des Ehepaares 27 Mio. Gulden als Gegenleistung für die Tatsache, daß sie auf die Erbschaft verzichteten. ${ }^{4}$

Womit beschäftigte - und beschäftigt sich noch immer - das Unternehmen 'Center Parcs'? Nachdem Derksen Ende der 40er Jahre angefangen hatte, sein Glück im Unternehmertum zu suchen, gründete er 1953 in Rotterdam das 'Sporthuis Centrum', ein Geschäft, später Großgeschäft mit vielen Filialen, für Sport und sonstige Freizeitartikel; 1978 verkaufte er diesen Zweig seiner Geschäfte mit insgesamt 20 Filialen. Er beschränkte sich in Zukunft auf den Betrieb von Parkanlagen mit Ferienwohnungen, die man wochenweise mieten kann und wobei der Urlaubsgast für seine Familie außerdem das Recht bekommt, die zentralen Einrichtungen zu benutzen (mindestens ein Luxusschwimmbad, Läden, verschiedene Restaurants, Sportanlagen usw.). Diese von Derksen entwikkelte Ferien-Formel war weltweit einzigartig, bis sie ebenso weltweit nachgeahmt wurde.

In den letzten 20 Jahren waren diese relativ preiswerten Feriendörfer bei steigendem Wohlstand und Kürzung der Arbeitszeit gerade in den Niederlanden und später ebenso in Frankreich, Großbritannien und Belgien sehr gefragt. Einige Zahlen dürfen den Erfolg dieses 1967 sehr bescheiden, mit einem Campingplatz begonnenen Unternehmens erläutern. ${ }^{5}$ In den 13 Niederlassungen verbrachten Ende der 80er Jahre insgesamt 2 Mio. Menschen einen Teil ihrer Freizeit. 1988 wurde $472 \mathrm{Mio}$. Gulden investiert. Der Umsatz betrug 1983185 Mio. Gulden, 1989 war von 500 Mio. Gulden die Rede; die Zahl der Mitarbeiter und 
Mitarbeiterinnen der Luxusbungalow-Parks in den genannten Ländern war 1989 auf 5000 gestiegen. Als Nettogewinn wurden 1983 fast 14 und 1988 fast 50 Mio. Gulden erzielt. Kurz nachdem das Sporthuis Centrum 1986 sehr erfolgreich auf der Börse introduziert worden war (Nettogewinn der Emission neuer Anteile: 60 Mio. Gulden), wurde der Konzern zwischen Herbst 1986 und Sommer 1987 umbenannt in 'Center Parcs NV'. Gerüchte über ausländische Käufer tauchen seitdem immer wieder im Wirtschaftsteil der Zeitungen auf.

Obwohl Center Parcs nach außen ein 'normales' Geschäft blieb,war für Derksen das Unternehmen jetzt nur ein Mittel, um seinen ideellen Aktivitäten über die Stiftungen die benötigten Gelder zu beschaffen. Sein Lebenszweck hatte sich 1982 geändert: Er möchte nur noch das Evangelium verbreiten und Not in der Dritten Welt lindern. Seine neue Einstellung wurde 1984 durch eine Veröffentlichung in der auflagenstärksten Zeitung der Niederlande, 'De Telegraaf, publik.

1989 verkaufte Derksen die Mehrheit (60\%) der Anteile seiner Aktiengesellschaft. Käufer war Scottisch \& Newcastle Breweries, ein Brauereikonzern in Edinburg. Kaufpreis: 667 Mio. Gulden. Der Gesamtwert des Unternehmens betrug 1989 also eine Milliarde Gulden. Durch eine Emission neuer Anteile bekam der Besitzer der Mehrheit der Anteile tatsächlich $65 \%$ der Anteile für eine Gesamtsumme von 747 Mio. Gulden. Das Ehepaar Derksen schenkte dem Personal insgesamt 3 Mio. Gulden (ungefähr 600 Gulden pro Person), zog sich aus der Direktion des Unternehmens zurück und bekam eine Beraterrolle angeboten. Tochter Trudie blieb weiterhin als Adjunkt-Direktor aktiv in dem Unternehmen. Ende September 1990 hatte Scottish \& Newcastle Breweries ihr Mehrheitsinteresse bis auf $80 \%$ aufgestockt. Nach der rapiden Expansion und der rechtzeitigen Transaktion von 1989 ergab sich übrigens die Notwendigkeit einer tiefgreifenden Umstrukturierung des Konzerns. Der Gewinn im ersten Halbjahr 1990 betrug 22,1 Mio. Gulden. Am 20.09.1990 gab der britische Konzern bekannt, ein öffentliches Gebot auf alle noch auf der Amsterdamer Effektenbörse gehandelten Anteile und Obligationsanleihen tun zu wollen.

\section{Lumen 2000 im Dienste der weltweiten Evangelisation}

Jährlich werden über Derksens Stiftung zum Zeugnis der Liebe Gottes durchschnittlich 3 Mio. Gulden in Dritte-Welt-Projekte, 5 Mio. Gulden in Evangelisations- und Zurüstungs-Projekte und 6 Mio. Gulden in Medien-Projekte gesteckt. Zusätzlich gibt es noch einmalige Spenden, z. B. 20 Mio. Gulden für die Priesterausbildung in der Dritten Welt und - im Jahre 1987 - 2 Mio. Gulden für die Veranstaltung von Massen-Exerzitien für 7000 Priester im päpstlichen Audienzsaal des Vatikans. Insgesamt 35 Mio. Gulden wurden 1989 katholischen Zwecken zur Verfügung gestellt. ${ }^{6}$

Daß ein Teil des Vermögens der Stiftung zum Zeugnis der Liebe Gottes für die Lösung der Probleme des Hungers, der Ungerechtigkeit und der Unterentwicklung in der Welt freigemacht wird, daß bei vielen einmaligen Projekten geholfen wird usw., bleibt hier unbesprochen. Ich beschränke mich auf den Medienbereich. Seit Oktober 1985 sendete die Stiftung während eines Jahres 


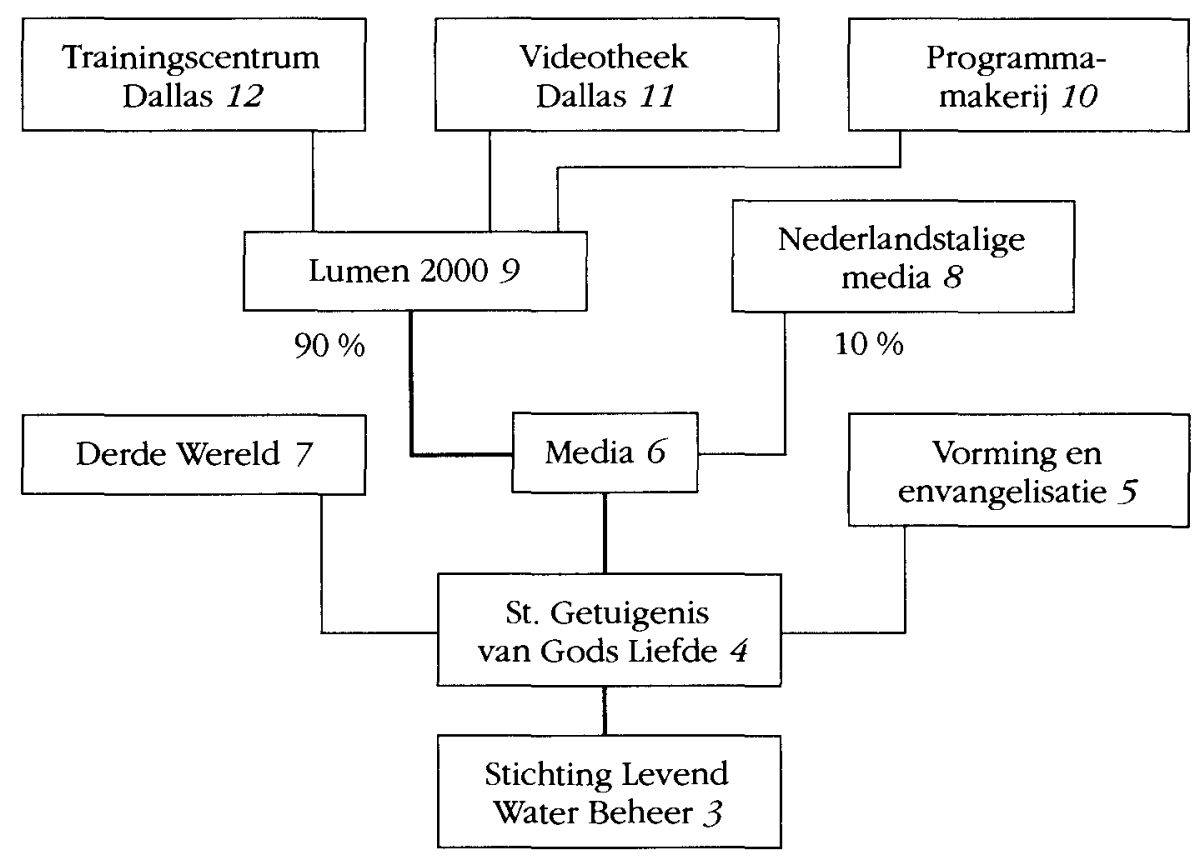

(Quelle: Tageszeitung TROUW vom 05.07.1989; für CS aktualisiert)?

vier ausländische religiöse Fernsehprogramme über den ersten europäischen Fernsehsatelliten. Die Einstellung dieser Sendungen hängt wahrscheinlich mit einer weltweiten Initiative Derksens zusammen: Er hatte größere Pläne, auch außerhalb des eigenen Kontinents.

Am 01.08.1986 veröffentlichten niederländische Zeitungen eine Pressemitteilung über die Gründung einer neuen katholischen Organisation in Dallas (USA) 'Lumen 2000'. Sie soll der Evangelisation der Welt dienen und mit dem Werbeslogan 'God will work for us' arbeiten. Einer der fünf Mitglieder des Vorstandes war Derksen, gleichzeitig Vorsitzender der 'Stiftung zum Zeugnis der Liebe Gottes'. Als Zielsetzung wurde erwähnt: Ein Gegengewicht bilden mit religiösen Sendungen in Hörfunk, im Fernsehen und auf Kassetten, die in Lateinamerika, Asien und Afrika darauf abzielen, die christliche Botschaft zu verbreiten, meistens auf einer fundamentalistisch-amerikanischen Grundlage. ${ }^{8}$

Als Mitbegründer wurde später der Amerikaner Bobbie G. Cavnar, ein ehemaliger Oberst der Luftwaffe, genannt. ${ }^{9}$ Cavnar war beteiligt an der Entwicklung des Spionageflugzeugs U-2 und der interkontinentalen Atomrakete. Während seines Ruhestandes verdiente er ein Vermögen im Geschäftsleben in Dallas und wurde Leiter der charismatischen Gruppe 'Catholic Community of God's Delight'. ${ }^{10}$

In Dallas verfügt Lumen 2000 für alle Teilnehmer in einem schon bestehenden katholischen Medienzentrum über ein Riesenbildarchiv mit einzelnen 
Bildern und kompletten Programmen (in mehreren Sprachen untertitelt); dort gibt es auch die modernste audiovisuelle Apparatur, um eigene Programme zu erstellen und angebotene Programme auszubessern. Schließlich befindet sich in Dallas das internationale Trainingszentrum, wo Studenten aus der ganzen Welt für Berufe im Bereich des Hörfunks und des Fernsehens ausgebildet werden. Die sich in der Aufbauphase befindende neue katholische Universität in Bogota bekommt eine von Lumen 2000 finanzierte Medienfakultät. Ein Studiengang für „Evangelisten“, die bei der Verbreitung des Glaubens Medien einsetzen, fing bereits 1989 an.

Beabsichtigt wird, noch vor Ende des Jahres 1990 wöchentlich ein internationales Fernsehprogramm anzubieten. „Berufungsfernsehen“ soll es sein, und Menschen in Lateinamerika, Afrika und Asien sowie - unter Einfluß der aktuellen Entwicklungen - auch Osteuropa sind die Zielgruppe. Derksen möchte Jesus Christus 'zu seinem 2000. Geburtstag ein Geburtstagsgeschenk machen: eine christliche Welt'. Die (Wieder-)Christianisierung der Welt sei möglich, davon ist er überzeugt, und zwar mit Einsatz aller technischen Kommunikationsmittel, die es schon gibt und die es noch geben wird. Neben Derksen und Cavnar gibt es als dritten Sponsor für Lumen 2000 den Finanzier des Eurotunnels und Großanteilhaber des Konsortiums für diesen Tunnel, Jean-Loup Dherse.

Lumen 2000 als Dach-Organisation katholischer Video- und Fernseh-Produzenten hat, laut einer Mitteilung der Stiftung zum Zeugnis der Liebe Gottes, Partner in mehr als 50 - manchmal wird sogar 70 als Zahl angegeben - Ländern, vor allem in der Dritten Welt. Angefangen wurde mit Ausbildungs-, Dokumentations- und Organisationsaufgaben; die programmatische Arbeit hinkte ein wenig nach und entwickelt sich erst seit dem dritten Jahr des Bestehens, seit 1988. ${ }^{11}$ Der Vorstand ist zusammengesetzt aus Vertretern jedes Kontinents, die meistens an der Spitze der internationalen charismatischen Bewegung stehen. Diese Mitglieder des Vorstands sind die Botschafter von Lumen 2000 bei den nationalen Episkopaten; sie schlagen auch die Projekte im Hauptsitz in Eindhoven vor. Wichtig ist auch das Lumen 2000-Büro in Rom, das die Beziehungen mit den vatikanischen Institutionen und Personen pflegt. Der Vatikan machte die insgesamt 117 päpstlichen Nuntiaturen in einem Empfehlungsbrief auf Lumen 2000 aufmerksam.

Das in Eindhoven veröffentlichte 'Lumen 2000 TV Magazin' begleitet das Pilotprojekt für das Fernsehprogramm, welches weltweit von über 80 Fernsehstationen ausgestrahlt wird: eine halbe Stunde pro Woche in Form eines Fernsehmagazin mit weltweiten Ereignissen aus der katholischen Kirche, zusammen mit kürzeren Sendungen über Gebet und Verkündigung. Die offizielle Lehre der Kirche ist Grundlage und Kriterium für den Inhalt der Sendungen.

Lumen 2000 versucht programmatische Mitarbeit aus allen Ecken der Welt zu bekommen und zeigt sich in Aufrufen 'particulary interested in news events with a Catholic aspect, local Catholic customs, and human interest stories including parish life and personal testimony'. ${ }^{12}$ Die Stiftung Gabriel in Hilversum ist die niederländische Organisation für die Zusammenarbeit mit Lumen 2000 und fungiert faktisch als Lumen 2000 Nederland. ${ }^{13}$ 


\section{Unterstützung für eine elektronische Kirche}

Die Medienevangelisation hat auch außerhalb von Lumen 2000 Osteuropa als Ziel entdeckt. Mit finanzieller Unterstützung der Stiftung in Eindhoven wird 'Eternal Word Netword Television' 1991 anfangen, aus Rom Hörfunkprogramme in sieben Sprachen für Hörer in Osteuropa und Nordafrika zu senden. Das Network ist die Initiative einer amerikanischen Äbtissin der Abtei der sog. 'Armen Klarissinnen' in Birmingham, Alabama. Mutter Angelica gründete 1981 mit nur 200 Dollar das erste katholische Satellitenfernsehen, baute ein eigenes Fernsehstudio hinter der Abtei, kaufte einen Ü-Wagen für Live-Übertragungen via Satellit, nannte ihn 'Gabriel' und macht seit 1987 rund um die Uhr christliches Fernsehen im amerikanischen Stil. Über 165 Kabelnetze in 37 Staaten der USA versucht Mutter Angelica inzwischen 14 Mio. Haushalte zu erreichen. Zweimal pro Woche bringt die Äbtissin eine eigene Show, 'Mother Angelica Live'. ${ }^{14}$

In den Niederlanden mit einer auch in Sachen Religion eher nüchternen Bevölkerung, wo man in den 80er Jahren nicht aufhörte, sehr kritisch und ablehnend über die Entwicklung der elektronischen Kirche in der USA zu berichten, ${ }^{15}$ las man derartige Geschichte in Zeitungen unterschiedlicher Gesinnung nicht ohne Kopfschütteln. Als der „Fernseh-Pfarrer“ Jim Bakker sein religiöses Millionenimperium 'PTL' (Praise the Lord/People that Love) und sich selbst 1987 in den Untergang stürzte, berichtete die niederländische Presse alle 'ins and outs'. Dies wiederholte sich, als Ende 1989 ein Buch über die Skandalgeschichte erschien. ${ }^{16}$ Hermann Boventer kennzeichnete neuerdings auch das bundesdeutsche Urteil über die 'sogenannten amerikanischen Fernsehverhältnisse' als 'generell nicht wohlwollend'. Laut Boventer werden in der Berichterstattung und in besonderem Maße von entsprechenden Programmen der öffentlich-rechtlichen Rundfunkanstalten die Fernsehapostel „mit ihren Unterhaltungskünsten als Scharlatane und Schwindler hingestellt, sie trivialisieren die Religion und machen aus ihr ein gewinnbringendes Geschäft“ ${ }^{17}$

Anfang 1987 wurde Derksen Mitglied des Verwaltungsrats des neuen Fernsehproduktionszentrums im Vatikan. In der italienischen Presse tauchen Meldungen über gute Beziehungen zwischen den drei genannten Herrn und dem Vatikan und/oder Papst Johannes Paul II. immer wieder auf. ${ }^{18}$ Mit dem amerikanischen Redemptoristenpater Tom Forrest, der seit 1986 in Rom Direktor der Verkündigungskampagne 'Evangelization 2000' ist, steht die 'Stiftung Zeugnis der Liebe Gottes' auch in Verbindung. Jedenfalls werden Erfahrungen ausgetauscht. ${ }^{19}$ Forrest wird übrigens auch als einer der Initiativnehmer der Organisation Lumen 2000 genannt.

\section{Frihe Initiativen in den Niederlanden}

Am 04.10.1983 erschien die erste Nummer einer konservativ-katholischen Zeitung, 'Katholiek Nieuwsblad', als Initiative einiger Laien (in doppelter Hinsicht), vereint in der 'Sint Arnulfusstichting' (St. Arnulfus-Stiftung). Obwohl ein tägliches Erscheinen beabsichtigt wurde, erscheint die Zeitung noch immer zweimal pro Woche. Die Zahl der Abonnenten ist stecken geblieben bei 20.793 Exemplaren (Stand: 01.01.1990) und einem Kioskverkauf von 1.450 Exemplaren, also insgesamt 22.243 Exemplaren; 1985 betrug die verkaufte Auflage insge- 
samt 16.500 Exemplare, davon waren 15.500 Abonnenten. ${ }^{20}$ Das 'authentischkatholische' Blatt hat im Vergleich mit den Tageszeitungen in Hinblick auf Vollständigkeit der allgemeinen Berichterstattung auf dem niederländischen Pressemarkt eine außerordentlich schwache Position. Gemäßigt-konservative Katholiken stören sich daran, daß die Redaktion, wenn sie Kritik an Personen übt, nur schwer den richtigen Ton zu finden weiß. Sogar die eher konservativen Bischöfe sind nicht immer glücklich über die Art und Weise, wie im 'Katholiek Nieuwsblad' polemisiert wird. Toleranz den vermeintlichen (progressiven) Feinden der Kirche gegenüber ist selten. Derksen unterstützte das Blatt in den ersten Jahren seines Bestehens; um ohne Verluste erscheinen zu können, hätte man mindestens 25.000 Exemplare verkaufen müssen. Wieviel Geld Derksen der Zeitung insgesamt zukommen ließ, ist nicht bekannt. Aus öffentlichen Mitteln empfing sie 1985 jedenfalls über den von der niederländischen Regierung ins Leben gerufenen 'Bedriifsfonds voor de Pers' (Betriebsfonds für die Presse) 650.000 Gulden.

Schon länger äußerte sich Derksen kritisch über den Nachwuchsmangel bei katholischen (d. h. konservativ-katholischen) Journalisten. Dies gilt auch für seine Haltung zur 1925 gegründeten Stiftung Katholische Rundfunkorganisation (Stichting Katholieke Radio Omroep, KRO) der kirchlichen Hierarchie. Den Journalisten der KRO machte er den Vorwurf, ihre katholischen Aufgaben nicht mehr richtig zu verstehen, als es zu einem öffentlichen Zusammenstoß zwischen Derksen und der KRO kam. Die KRO-Berichterstattung 1985 zum Papstbesuch in den Niederlanden hatte ihm überhaupt nicht gefallen. Bei der Eröffnung des Marienjahres durch Papst Johannes Paul II. stellte Derksen der KRO die Möglichkeit zur Live-Übertragung eines Gebetsgottesdienstes mit dem Papst am 06.06.1987 via Satellit zur Verfügung. Die KRO lehnte das Angebot ab, das in der Presse als 'relivisie' ('Relivison': Religion und Television) abgetan wurde ${ }^{21}$ Sie sendete am nächsten (Sonntag-)Morgen nur eine Zusammenfassung. In Maastricht konnte man auf einem im Freien aufgestellten Großbildschirm den Gottesdienst verfolgen. 12 Orte, an denen Maria verehrt wird, wurden in einer Sendung für 1,5 Milliarden Zuschauer unter Einsatz von 18 Satelliten miteinander verbunden. Die Kosten des 'Global Media-Projekts', 5. Mio. Gulden, wurden getragen von Global Media (USA), Lumen 2000, dem französischen Produzenten der BIC-Kugelschreiber und von kleineren Spendern. ${ }^{22}$

\section{Eine römisch-katholische Rundfunkorganisation?}

Derksen zeigte sich mit der Ersatzlösung im Rahmen des Global MediaProjekts nicht zufrieden und versuchte noch 1987, eine konservative römischkatholische Rundfunkorganisation (Rooms-Katholieke Omroep, RKO) als Gegengewicht zur katholischen Rundfunkorganisation KRO zu gründen. (In den konservativen Kreisen in den Niederlanden legt man sehr viel Wert auf die Feststellung, daß in den 60er Jahren in den Niederlanden das Adjektiv 'römisch' verloren gegangen sei.) Derksen erlitt mit seinem RKO-Projekt eine schwere Niederlage. Die vier konservativen Bischöfe der Niederlande und namentlich der Erzbischof Adrianus Kardinal Simonis (Utrecht) machten nicht mit und bevorzugten den Versuch, die KRO auf die Dauer wieder auf die Linie des Episkopats zu bringen. 
Gesetzlich bekommen die Kirchen in den Niederlanden Sendezeit und die dafür notwendigen Mittel eingeräumt. Die katholische Kirche bekommt pro Woche 62 Minuten Fernseh- und 180 Minuten Hörfunksendezeit; der Episkopat stellt die Sendezeit und die Mittel der KRO zur Verfügung. Als Derksen mit seinem Plan für eine konservativ-katholische Rundfunkorganisation an die Öffentlichkeit trat, hatte der Episkopat gerade zum erstenmal mit der KRO Richtlinien für das Mitspracherecht der Bischöfe in bezug auf die Programminhalte dieser kirchlichen Sendezeit verabredet. Außerdem muß man feststellen, daß gerade die KRO sich in den letzten 20 Jahren als einziges noch bestehendes katholisches Massenmedium dieses Umfangs um ihre katholische Identität kümmerte und dabei Polarisierungen zwischen den Katholiken zu vermeiden versuchte. Derksen erntete mit seiner Initiative zwar große Aufmerksamkeit in der Presse ${ }^{23}$ er mußte dennoch vor den Bischöfen kapitulieren, die - wie er behauptete - von der KRO nicht gut behandelt worden waren. Innerhalb einer Woche sah Derksen sich gezwungen, sein Vorhaben in den Schubladen verschwinden zu lassen.

Am 15.06.1987 machte NRC Handelsblad die Öffentlichkeit noch einmal aufmerksam auf die Pläne des Frühjahrs; ein Sprecher der 'Stiftung zum Zeugnis der Liebe Gottes' weckte den Eindruck, noch mit der Vorbereitung beschäftigt zu sein, obwohl Derksen es schon besser wußte. Er hatte sich geirrt in bezug auf die bischöfliche Zustimmung. Nicht nur Derksen, sondern auch die KRO blieb dem Episkopat treu gesinnt. In der neuen, am 01.01.1990 in Kraft getretenen Satzung der am genannten Datum zu einem Verein umgewandelten KRO (mit zu diesem Zeitpunkt 626.134 Mitgliedern) heißt es in Absatz 2: „Der Verein hat die Aufgabe, Hörfunk und Fernsehen zu betreiben zu Orientierung, Information und Unterhaltung der Hörer und Fernsehzuschauer. Bei der Erfüllung ihrer Aufgabe anerkennt und bekennt er die Offenbarung Gottes, wie diese von der katholischen Kirche bewahrt und weitergegeben wird." 24 Die katholische Rundfunkorganisation bemüht sich, im Dienste der ganzen katholischen Gemeinschaft und nicht nur für den progressiven oder konservativen Teil der katholischen Bevölkerung zu funktionieren. Daß herkömmliche Publizistikstrukturen in einer pluralistischen Gesellschaft wie der der Niederlande noch immer leicht unter Druck und sogar in eine Krise geraten können, läßt sich verstehen. ${ }^{25}$

Abgesehen von der Frage, ob Derksens Neugründung im Rahmen des am 01.01.1988 in Kraft getretenen Mediengesetzes Chancen gehabt hätte, hätte eine neue konservativ-katholische Rundfunkorganisation jedenfalls die Spaltung der niederländischen Katholiken in zwei Strömungen besiegelt, und dafür mochten die Bischöfe keine Mitverantwortung tragen. In Kommentaren und Hintergrundanalysen der Tageszeitungen wurde das Dilemma des Episkopats unmißverständlich dargestellt.

Anfang 1990 gab es wiederum Reibungen zwischen der KRO und - diesmal - Lumen 2000. Der niederländische Medienbischof Msgr. Philip Bär OSB (Bischof von Rotterdam) zeigte sich enttäuscht, als die KRO es ablehnte, das erste von Lumen 2000 produzierte und kostenlos angebotene Fernsehprogramm zu senden. Dieses Programm mit dem Titel 'Miracles of the Eucharist' behandelt 4 der insgesamt 165 Wunder aus der Geschichte und war, so meinte 
der für Medienfragen zuständige Bischof, der selber in der Sendung eine Rolle spielte, auch sehr geeignet für das niederländische Publikum. Die Leitung der KRO war jedoch anderer Meinung: die Produktion widmete dem Erleben der niederländischen Gläubigen zu wenig Aufmerksamkeit. ${ }^{26}$ Msgr. Bär zeigte für dieses Argument Verständnis, nicht aber Lumen 2000.

Das gedruckte Wort ließ die 'Stiftung zum Zeugnis der Liebe Gottes' inzwischen ebenso wenig unberührt wie die elektronischen Medien. Seit Herbst 1987 erscheint $12 \mathrm{Mal}$ pro Jahr als katholisches Monatsheft 'het familieblad MANNA', eine Illustrierte, die an die vor 20 Jahren in den Niederlanden abgeschlossene Zeit der herkömmlichen Familienwochenblätter erinnert. Obwohl technisch hervorragend im Vierfarbendruck gemacht, bleibt der Inhalt ziemlich einseitig Erinnerungen an eine heile Welt vorbehalten. In Fragen des Schwangerschaftsabbruchs, der Euthanasie, Ehescheidung usw. wird systematisch die orthodox-katholische Lehre hervorgehoben und die bestehende reale Situation verneint oder gerügt. Aktivitäten und Schwerpunkte der 'Stiftung zum Zeugnis der Liebe Gottes' erhalten die besondere Aufmerksamkeit der Redaktion. In Interviews mit bekannten Sängerinnen, Politikern usw. wird nur das Positive angesprochen, Probleme scheinen nur dazu da zu sein, um durch den Glauben überwunden zu werden. Als Auflage wird für Manna 50.000 Exemplare angegeben. Ab dem 3. Jahrgang, Nr. 3, März 1990, übernahm Derksen selber die Funktion des Direktors. Kostenlos verbreitet wird das Magazin 'Getuigenis van Gods Liefde' (1990 im 5. Jahrgang, Auflage: 45.000 Exemplare). Im eigenen Verlag (Umsatz 1989: 200.000 Gulden) der 'Stiftung zum Zeugnis der Liebes Gottes' erscheinen außerdem Bücher, Broschüren usw. Über das Tochterunternehmen 'Effata' werden jährlich ungefähr 7.000 Tonkassetten mit Vorträgen und Liedern aus dem eigenen Repertoire verkauft. ${ }^{27}$

\section{Die Tragik einer Kirchenprovinz und die Folgen}

Die Situation des Katholizismus in den Niederlanden ist in den letzten Jahrzehnten bedauerlicherweise gekennzeichnet durch harte Auseinandersetzungen zwischen einer konservativen Strömung mit verschiedenen Gruppierungen einerseits und einer progressiven, ebenfalls pluriformen Strömung andererseits. Dazwischen entwickelt sich das Meer der Gleichgültigkeit, der Ablehnung der Kirche und der Erosion der Religiosität - und dies nicht nur bei der jüngeren Generation. Piet Derksen gehört zu einer Generation, die sich in den letzten Dezennien teilweise im konservativen Lager verschanzte. Wieso es soweit kommen konnte, muß näher erklärt werden. ${ }^{28}$

In einer großen Gemeinschaftsleistung haben es sich die Katholiken der Niederlande in der ersten Hälfte des 20. Jahrhunderts erkämpft, daß sie politisch, intellektuell und gesellschaftlich mit der nicht-katholischen Bevölkerung gleichziehen konnten. Als Folge der Reformation waren sie drei Jahrhunderte lang eine unterdrückte Minderheit gewesen. Bis zum Anfang der 60er Jahre waren die Katholiken von einem sehr starken Zusammengehörigkeitsgefühl gekennzeichnet, das eine unbeschränkte Bejahung der hierarchischen Gewalt der Bischöfe und Priester voraussetzte. 
Diese hier nur knapp geschilderte Konstellation hatte zwar große Vorteile, aber auch deutlich negative Seiten. Die kirchliche Gewalt förderte in diesen Jahrzehnten nicht nur die Verkündigung des Evangeliums und des Reiches Gottes, sondern zugleich die gesellschaftliche Emanzipation einer Bevölkerungsgruppe. Aber gerade durch die enge Zusammengehörigkeit war der soziale Druck auf das Individuum sehr stark. Die Einförmigkeit brachte für den Einzelnen eine bestimmte Unfreiheit mit sich.

In den 60er Jahren vollzog sich dann der große Zusammenbruch oder der große Durchbruch; je nachdem, wie man diese Zeit und ihre Entwicklungen beurteilt. Als Ursachen könnte man vieles nennen: Die Emanzipation war vollendet; die Katholiken waren auf keinem Gebiet mehr eine zurückgesetzte Gruppe, weder bildungsmäßig noch finanziell oder sozial. Der Sinn der allzu engen Zusammengehörigkeit ging damit verloren; immer mehr entdeckte man die Nachteile einer so stark betonten Autorität; die Unfreiheit des Einzelnen und die Einförmigkeit wurden immer stärker empfunden. Emanzipation bedeutete auch die Entwicklung von stark gruppengebundenen Menschen zu Erwachsenen mit einer eigenen Meinung und Auffassung.

Diese doppelt emanzipierten Katholiken begannen sich eine andere Art Kirche zu wünschen, wo die einzelnen Gläubigen in wechselseitig anerkannter Freiheit Gemeinde Christi sein können. In diese Zeit fiel das II. Vatikanische Konzil, dessen Ergebnisse mit Begeisterung aufgenommen wurden. Die 60er Jahre waren 10 Jahre religiöser Begeisterung und Experimentierens, wobei die niederländischen Katholiken ins Rampenlicht der internationalen Öffentlichkeit gerieten. ${ }^{29}$

Wo Meinungen und Überzeugungen klipp und klar geäußert werden können, entstehen leicht Meinungsverschiedenheiten; da entsteht dann links und rechts, progressiv und konservativ. Die Diskussion zwischen beiden wurde schwer und aussichtslos, weil die Progressiven erwarten, die Anderen mit neuen Argumenten zu gewinnen, während die Konservativen eigentlich nicht diskutieren wollen, sondern nur von ihren Gesprächspartnern fordern, daß diese die alten Wahrheiten bedingungslos akzeptieren. Die Periode seit 1970 ist eine Zeit der Abriegelung, der Polarisierung.

\section{Glaubwürdige Kommunikation fördern}

Der Einsatz der Medien zur Verkündigung, wie ihn Derksen als konservativer Katholik befürwortet und fördert, verdient eine kritische Dauerreflexion und Diskussion, die außerhalb dieses Aufsatzes weitergeführt werden soll. Wie realistisch oder wirklichkeitsfremd ist die Absicht, die Welt inklusive der Niederlande bis zum Jahr 2000 zu bekehren? Inwiefern wird theologisches und kommunikationswissenschaftliches Wissen mit der Praxis dieses Typs der Evangelisation konfrontiert? Was bedeutet diese Evangelisationsoffensive unter der Schirmherrschaft eines Papstes, der den Ruf des großen Kommunikators (big communicator) hat, für die Ökumene? Wird dem Ideal der herrrschaftsfreien Kommunikation dadurch gedient, daß man ausschließlich versucht, einseitig konservatives Gedankengut zu vermitteln? Bietet man wirklich das Manna als 
Brot des Zuges durch die Wüste oder an Stelle dieses Brotes einen Stein? Überschätzen Derksen und seine Mitarbeiter die Wirkung der Printmedien und der elektronischen Medien auf nationaler und internationaler Ebene nicht? Und wenn die Möglichkeiten richtig eingeschätzt werden, muß dann vorrangig den konservativen fundamentalistischen und evangelikalen Christen gedient werden? Der Aufstieg der evangelikalen und fundamentalistischen Fernsehprogramme in der Welt ist sowieso schon gesichert. ${ }^{30}$ Über Prioritäten sollte aber diskutiert werden dürfen.

Nur zwei Gedanken, Fragen eigentlich, möchte ich zu der m. E. notwendigen Auseinandersetzung beitragen. Erstens: Was bringt soviel Aufwand an Energie und Kapital für das gläubige Leben der Gemeinde als Quelle des Heils? Auch sympathische Medienprediger oder BTX-Seelsorger müssen, laut Eva Schüler-Petkewitz (Pressestelle Landeskirchenamt der Evangelischen Kirche Rheinland), damit rechnen, als 'Hausgeister' in den Wohnstuben eingesetzt zu werden. „Das ist“, meint sie, "nicht verboten und mag ganz amüsant sein. Es trägt aber nichts dazu bei, Gemeinde zu werden. Gemeinde ist ebensowenig zu definieren wie Kommunikation. Gemeinde ereignet sich. Sie ist im „Dazwischen" angesiedelt, das Menschen in ihrem Glauben an Gott verbindet. Dennoch kann sich die Gemeinde den Bildschirmen nicht entziehen. (...) Die Gemeinde wendet sich nicht ab, sondern sie lädt ein zum Erlebnis: Daß Gott bei uns ist, weil Jesus ganz mit uns lebt und wir ihn immer noch sehen, hören, fühlen, schmecken und riechen. Der Bildschirm kann das nicht zu verstehen geben. Aber er sollte eine Herausforderung sein, es anders zu versuchen und anderwo: im alltäglichen 'Dazwischen' und nicht nur mit Worten und Bildern, sondern mit allen Sinnen. “31

Zweitens: Was bedeutet die von konservativ-katholischen Kräften gesteuerte Medienarbeit für die Glaubwürdigkeit bei den Zielgruppen? Wenn wir, laut einer Studie des Ökumenischen Rates der Kirchen aus dem Jahr 1983, den Begriff Kommunikation ('grundlegender gemeinsamer Akt der Mitteilung von und Teilhabe an Information, um Leben anzuregen und eine Gemeinschaft aufzubauen') nehmen und diesen Prozeß mit dem Adjektiv 'christlich' belegen, was passiert dann? Antwort: 'Dann muß christliche Kommunikation offen dafür sein, sich mit dem gesamten Leben und der ganzen Schöpfung zu beschäftigen. Ihre Aufgabe wird es sein, in allem, was irgend jemand im Sinne des Guten und der Liebe, für die Wahrheit, die Gerechtigkeit, die Schönheit und den Frieden tut, ob nun eingestandenermaßen oder nicht, die Gegenwart und das Wirken Christi zu erkennen. Christliche Kommunikatoren sind aufgerufen, diese Gegenwart zu bezeugen'.$^{32}$ Diese Stellungnahme ist aktueller als je zuvor.

Hoffentlich wird man in von Derksen beherrschten Kreisen und von ihm ins Leben gerufenen Organisationen diskussionsfreudiger. Es kann doch nicht so sein, daß sie innerhalb eines Jahrzehnts, im von ihnen so stark betonten Jahr 2000, auf einen gescheiterten Versuch, die Welt zu bekehren, zurückblicken müssen, einfach weil man unrealistisch, d. h. an den Bedürfnissen der Menschen vorbei, Schmalspur gefahren ist. Für Geld kann man in der Welt des ausgehenden 20. Jahrhunderts viele materielle und immaterielle Versprechungen kaufen. Die Bekehrung der Menscheit zur sog. 'guten alten Zeit' bedeutet 
jedoch eine Regression, wofür es eigentlich keine Mittel geben dürfte, wenn man die Prioritäten der christlichen Kommunikation gewissenhaft überprüft. Den Stein der Weisen zu finden, ist zwar nicht einfach, einen Stein des Anstoßes könnte man jedoch mindestens zu vermeiden versuchen. ${ }^{33}$

\section{Anmerkungen:}

1 Anschrift des Instituts: 32, rue de l'Association/Verenigingsstraat 32, B-1000 Brussel, Belgien, 'Managing director' ist der Belgier Marcel Bauer, der während seines vielseitigen Werdegangs im Medienbereich $u$. a. bei MISSIO (Aachen, BRD) tätig war. 'Academic director' ist der Franzose Dr. Bernard de Castera, der u. a. in Angers (Frankreich) beteiligt war an der Ausbildung für Kommunikationsberufe.

2 Die Fernsehjournalisten Aaltje van Valderen und Lejo Schenk stellten für das niederländische Fernsehmagazin 'Kenmerk' eine Dokumentation mit dem Titel 'Geschenk für einen 2000-jährigen' zusammen. Im bundesdeutschen Fernsehen wurde am 12.05.1989 'Der Satelliten-Jesus. Bekehrung per Bildschirm', eine Dokumentation von Martin Blachmann unter Redaktion von Werner Hamerski, gesendet. In dieser Sendung des WDR wurde Piet Derksen behandelt in Zusammenhang mit Lumen 2000 und der Zielsetzung, Jesus zu seinem 2000. Geburtstag ein besonderes Geschenk zu machen: eine evangelisierte Welt, bekehrt durch das Fernsehen.

3 Seit 1965 wird die Persönlichkeit und das Wirken Robert Schumans in der Bundesrepublik Deutschland gewürdigt durch den Robert-Schuman-Preis für wissenschaftliche und publizistische Leistungen im Rahmen der europäischen Einigung.

4 Henri Stolwijk, 'Sporthuis Centrum slaat vleugels uit in buitenland', in: Elseviers Weekblad vom 07.06.1986, 19.

5 Die finanziellen und organisatorischen Informationen wurden den vielen Zeitungsausschnitten in meinem Archiv entnommen; am besten informiert zeigten sich NRC Handelsblad am 08.06.1988, am 04. und 05.07.1989 und am 06.02.1990, Trouw am 05.07.1989, insbesondere der Aufsatz von Fernsehredakteurin Aaltje van Valderen am 18.08.1989, de Volkskrant am 12.08.1989 und Quote, Juli/August 1989, 41-45.

6 Diese Zahlen wurden NRC Handelsblad vom 27.07.1989 entnommen.

73 = Stiftung Lebendiges Wasser Verwaltung

$4=$ Stiftung Zeugnis der Liebe Gottes

$5=$ Zurüstung und Evangelisation

$6=$ Medienarbeit

$7=$ Dritte Welt-Projekte

$8=$ niederländischsprachige Medien

$9=$ Lumen 2000

$10=$ Programmgestaltung

$11=$ Videothek Dallas

12 = Trainingscentre Dallas

8 Meldung u. a. in der Tageszeitung 'De Gelderlander' vom 01.08.1986.

9 In der Tageszeitung 'Het Parool' vom 24.10.1989.

10 Jan van der Putten behauptet in der Tageszeitung 'De Volkskrant' vom 29.10.1988, daß Derksen an der charismatischen Gemeinschaft der Brüderschaft Jesu beteiligt sei.

11 'Lumen 2000 International', in: Getuigenis van Gods Liefde, 3/1988, Nr. 2, 10.

12 Lumen 2000', in: Cine \& Media 10/1989, Nr. 4, 2.

13 'Lumen 2000 Nederland', in: Getuigenis van Gods Liefde 3/1988, Nr. 2, 11

14 Interview mit Mother Angelica, in: Manna, März 1990, 23-25.

$15 \mathrm{Vgl}$. z. B. Henk Kempes, 'de elektronische Kerk als evangelisatiebeweging', in: Hoan Hemels und Henk Hoekstra, Media en religieuze communicatie. Een uitdaging aan de christelijke geloofsgemeenschap, Hilversum 1985, 249-258.

16 Charles E. Sheppard, The rise and fall of Jim Bakker and the PTL ministry, Atlantic Monthly Press 1989. 
17 Hermann Boventer, 'Elektronische Kirche in den USA. Hat sie sich zu Tode amüsiert?', in: Rolf Terheyden (Hg.), Beruf und Berufung. Zweite Festschrift für Johannes Binkowski, Mainz 1988, 138-146, 139. Boventer plädiert für einen Abbau der Vorurteile und Stereotypen in Bezug auf das Phänomen der elektronischen Kirche.

18 Siehe Anm. 9.

19 Z.B. während einer Tagung, der 'neuen Evangelisation' gewidmet, am 16.11.1989; ein Bericht wurde im (konservativen) Bistumsblatt der Diözese Den Bosch vom 25.11.1989, 5, veröffentlicht. Dieses 'Bisdomblad' zeigte sich öfter gut informiert über Vorhaben und Aktivitäten der 'Stiftung zur Zeugnis der Liebe Gottes', auch in Bezug auf Medienarbeit.

20 Die Zahlen wurden der Fachzeitschrift der niederländischen Joumalisten 'De Journalist' vom 21.05.1990, 38, entnommen.

21 Sehe Henk van Gelder, in: NRC Handelsblad vom 15.06.1987 und Gerard Geradts in de Volkskrant vom 26.05.1987.

22 Die christliche Tageszeitung 'Trouw' fürchtete in einem Kommentar vom 09.06.1987 eine negative Auswirkung der Sendung auf die Ökumene in den Niederlanden und 'De Volkskrant' vom 06.06.1987 erläuterte, wie Global Media diese religiöse Riesenshow der Kommunikationstechnik vermarktete und sich Einnahmen aus der Verkauf der Übernahmerechte, der Filmaufzeichnungen und Tonkassetten, Bücher usw. sicherte.

23 Die neutral-konservative Tageszeitung 'De Telegraaf brachte die Meldung am 08.05.1987 als Aufmacher, angeblich von Derksen und/oder seiner Stiftung durch 'news-management' beeinflußt. Die viele Presseausschnitte und Reaktionen darauf in meinem Archiv sind ein Beleg dafür, wieviel Unruhe Derksen und seine Stiftung für kurze Zeit stifteten.

$24 \mathrm{Zit}$. aus der Broschüre 'Statuten en hiushoudelijk reglement [voor de vereniging KRO], Hilversum O. J., 7.

25 Vgl. in diesem Zusammhang Michael Schmolke, 'Pluralistische Publizistikstrukturen und ihre Probleme', in: Joan Hemels und Michael Schmolke (Hg.), Katholische Publizistik in den Niederlanden, München/Paderborn/Wien 1977, 7-14.

26 Lt. Harrie Smeets, 'Bär-2000', in: De Bauzin vom 23.02.1990, 10-11, zahlt Lumen 2000 den Lieferanten eines Programmsegments (Dauer 5 Minuten) 1.000 \$. Diese 'evangelic segments from all continents' und 'special programs'werden in Hilversum, Niederlande, jede Woche zu einem Programm zusammengeschmiedet.

27 Diese Daten verdanke ich Aaltje van Valderen, Fernsehredakteurin des Magazins 'Kenmerk', das die Dokumentation über Derksen und sein Evangelisationsvorhaben sendete. Sie veröffentlichte einen Aufsatz über das Thema in der Tageszeitung 'Trouw' vom 05.07.1989.

28 Ich stütze mich hierbei u. a. auf den Text eines nicht veröffentlichten Vortrags von Lamber van Gelder, Nijmegen, Niederlande, gehalten im Rahmen der Verlegerkonferenz der Arbeitsgemeinschaft Katholische Presse e. V., am 06.06.1984 in Essen.

29 Die Rolle der Intellektuellen und ihr Wirken in der Öffentlichkeit ist Thema einer Doppeldissertation: Ed Simons und Lodewijk Winkeler, Het verraad der Clercken. Intellectuelen en hun rol in de ontwikkelingen van het Nederlandse katholicisme na 1945, Baarn 1987.

Deutsch- und englischsprachige sozialwissenschaftliche Aufsätze zur Orientierung sind: Jan Thurlings, 'Pluralism and assimilation in the Netherlands, with special reference to Dutch catholicism', in: International Journal of Comparative Sociology 19/ 1979, 82-100; Albert Felling, Jan Peters und Osmund Schreuder, 'Identitätswandel in den Niederlanden', in: Kölner Zeitschrift für Soziologie und Sozialpsychologie 34/ 1982, 26-53; Albert Felling und Jan Peters, 'Der Säkularisierungsprozeß in den Niederlanden zwischen 1966 und 1985, in: H. O. Luthe und H. Meulemann (Hg.), Wertwandel - Faktum oder Fiktion?, Frankfurt/M. 1988, 98-139.

$30 \mathrm{Vgl}$. das Standardwerk über das christliche Fernsehen von Peter Horsfield, Religious Television. The American experience, New York 1984 und Konrad Ege, "Gott wollte, daß Reagan gewählt wird ...". Das christliche Fernsehen in den USA', in: Medium 16/1986, Nr. 2, 6-12. Mehr Literaturangaben findet man bei Boventer, a. a. O., 146. 
31 Eva Schüler-Petkewitz, 'Christliche Kommunikation - ganzheitliche Kommunikation', in: Ulrich Pätzold und H. Gerd Würzburg (Hg.), Durchzug. Dortmunder Journalistik-Lesebuch, Kurt Koszyk zum 60. Geburtstag, Dortmund 1989, 128-140, 139.

32 Zit. aus 'Was ist glaubwürdige christliche Kommunikation? Eine Studie des Ökumenischen Rates der Kirchen, erarbeitet vom "Communications Department of the World Council of Churches"', in: Medium 13/1983, Nr. 7, 28-32, 28-29.

33 Das Manuskript wurde im September 1990 abgeschlossen.

\section{SUMMARY}

A Dutch multimillionaire, Piet Derksen, has been endeavouring to missionize world-wide by using all the publishing and electronic media at his disposal. He contributed his fortune to a foundation which has since then supported with large sums of money the evangelisation organisation 'Lumen $2000^{\circ}$ ' which has created a sensation internationally. The radio empire Lumen 2000 wishes to give Jesus Christ a special present for his two thousandth birthday: an evangelized world converted by television. Lumen 2000 is also supported by the Pope and the Vatican. Nevertheless the question arises how one has to evaluate this media work controlled by conservative forces; nominally in the light of creation of a Christian community full of life, and of the demand for a credible form of communication.

Not only at an international level but also in Holland Piet Derksen has tried to reverse the developments set in motion during the Second Vatican Council among Catholic people. The author explains this obdurate mobilization of the media in the service of conservative thought with the wide-spread uncertainty about standards and values. It offers fertile ground for efforts at regression. The author particularly criticizes as unhelpful Derksen's deliberately encouraged strengthening of conservative trends in the Dutch Catholic Church and does not expect much good to come from its effect at an international level.

\section{RÉSUMÉE}

Un multimillionnaire néerlandais, Piet Derksen, s'efforce, en se servant de tous les moyens (presse et médias électroniquesè) qui sont à sa disposition, de propager sa foi à l'échelle mondiale. Il a investi sa fortune dans une fondation qui, depuis, a soutenu financièrement l'organisation d'évangélisation "Lumen $2000^{\circ}$, qui fait sensation. Cet empire audiovisuel „Lumen $2000^{\circ}$ se propose de faire un don particulier à Jésus Christ lors de son 2000ième anniversaire: un monde évangélisé, converti par la télévision. „Lumen $2000^{\prime}$ peut compter sur le soutien du pape et du Vatican. Pourtant, la question se pose comment juger cette opération médiatique, dirigée par des forces conservatrices; ce jugement doit surtout tenir compte du développement de paroisses chrétiennes en plein essor et d'une communication digne de foi.

Ce n'est pas seulement au plan international mais aussi aux Pays Bas que Piet Derksen essaie d'annuler le mouvement initié par le concile de Vatican II, mouvement qui a saisi le peuple catholique. L'auteur explique cette mobilisation opinitre des médias au service de la pensée conservatrice par la désorientation largement répandue au sujet des normes et des valeurs. Cette désorientation offre un terrain favorable aux tendances de régression. L'auteur caractérise comme peu utiles les efforts délibérés de Derksen pour renforcer les courants conservateurs dans l'église catholique des Pays Bas, et il craint, de ces actions internationales, des résultats négatifs.

\section{RESUMEN}

El multimillonario holandés Piet Derksen está haciendo ingentes esfuerzos por misionar en todo el mundo a través de todos los medios impresos y electrónicos a su disposición. El ha puesto toda su requeza en un Fundación que desde entonces financia, 
entre otras cosas, la organización para la evangelización 'Lumen 2000'. Organización que ha logrado alcanzar una considerable imagen internacional. El imperio radiotelefónico de Lumen 2000 quiere hacer un regalo especial a Jesuscristo para su cumpleanos 2000: Un mundo evangelizado, convertido al cristianismo a través de la televisión. Lumen 2000 es apoyada también por el Papa y el Vaticano. No obstante uno se hace la pregunta de cómo se puede juzgar este trabajo a través de los medios de comunicación dirigido por fuerzas conservadoras visto a la luz de la construcción de comunidades cristianas vivas y de su promoción a una credibilidad en la comunicación.

Piet Derksen intenda, no sólo a nivel internacional sino también a nivel de Holanda, hacer retroceder los rasgos de desarrollo en el pueblo católico echados a cominar por el Concilio Vaticano II. El autor explica la obstinada de los medios de comunicación al servicio del pensamiento conservador como reacción frente a la inseguridad, respecto a las normas y valores, extendida por todo el mundo. Esto ofrece un suelo fructifero para los anhelos de regresión. El autor juzga la exigencia de Piet Derksen de un mayor fortalecimiento de la corriente conservadora dentro de la iglesia católica holandesa, como poco efectiva y espera poco de su repercusión en el escenario internacional. 